\title{
Artículos
}

\section{Importancia de la población centroamericana nacida entre 1980 y 1999 sobre el futuro inmediato de la región}

\author{
José Arnoldo Sermeño Lima
}

\section{Resumen}

En estas páginas se identifica la importancia cuantitativa que jugará en el futuro inmediato la actual población infantil de Centroamérica y Panamá, asi como también aquella otra que nacerá durante la presente década. El análisis se centra en el impacto que esas generaciones tendrán tanto sobre el conjunto de la población como sobre la población en edad escolar y sobre la población económicamente activa.

\section{Introducción}

El objeto de este trabajo es identificar la importancia cuantitativa que jugará en el futuro inmediato la actual población infantil, así como también aquella que nacerá durante la década en curso. Esa importancia se analizará fundamentalmente en el impacto que esas generaciones tendrán tanto sobre el conjunto de la población, así como también sobre dos subpoblaciones: aquella en edad escolar y sobre la población económicamente activa. Es bien sabido que ambas juegan un rol importante en el desarrollo socio-económico de todas las sociedades.

El análisis se centrará en los paises del istmo centroamericano, tanto en forma individual como también en su conjunto. Con respecto a esto último, se utilizarán dos tipos de totales: al hacer referencia al conjunto de los cinco países que cons- tituyeron la antigua República Federal de Centro América, el todo se conceptualizará como "Centroamérica", o "C.A."; mientras que al incluir además a Panamá, se usará la acepción de "istmo".

2. Breve descripción de la situación demográfica centroamericana

El Cuadro 1 presenta una estimación de la población de cada país centroamericano para 1991. A partir de esa información, puede deducirse que la población del conjunto del istmo representa el 6.7 por ciento del total latinoamericano (CELADE, 1987a, p. 13). Guatemala, El Salvador y Honduras concentrarán ese año casi las tres cuartas partes de la población centroamericana (74.0 por ciento), y un poco más de los dos tercios de la del conjunto del istmo (67.8 por ciento).

Las elevadas tasas de natalidad y crecimiento 
que han privado históricamente en la región (CELADE, 1987) han tenido como consecuencia el generar una población constituida principalmente por efectivos jóvenes en cada país del istmo centroamericano. Debido a esta estructura, las poblaciones centroamericanas continuarán creciendo de manera acelerada en los decenios inmediatos, a pesar de haberse iniciado ya un franco proceso de reducción de la fecundidad en cada uno de los países del istmo (CELADE, 1987).

Con el transcurso del tiempo, esa reducción de la fecundidad conducirá a un relativo "envejecimiento" de esas poblaciones. Sin embargo, en las décadas inmediatas, la población joven demandará con urgencia nuevas facilidades educativas, de salud, empleo, alimentos, etc., que -de no tomarse urgentes medidas correctivas en el momento actual- vendrán a agravar el actual déficit existente en todos esos campos, con imprevisibles consecuencias sobre otros en los cuales también tengan incidencia.

\section{Cuadro 1}

1991: Población y estructura porcentual por edad de la población, por país

\begin{tabular}{|c|c|c|}
\hline País & & $\begin{array}{c}\text { Población } \\
\text { (miles) }\end{array}$ \\
\hline Guatemala & & 9,467 \\
\hline El Salvador & & 5,375 \\
\hline Honduras & & 5,298 \\
\hline Nicaragua & & 3,999 \\
\hline Costa Rica & & 3,088 \\
\hline Panamá & & 2,466 \\
\hline CA. & & 27,227 \\
\hline Istmo & & 29,693 \\
\hline \multicolumn{3}{|c|}{ Estructura por edades (porcentaje) } \\
\hline$<15$ & 15 a 59 & $60+$ \\
\hline 45.6 & 49.5 & 4.9 \\
\hline 45.3 & 49.2 & 5.5 \\
\hline 45.4 & 49.6 & 5.0 \\
\hline 46.2 & 49.5 & 4.3 \\
\hline 36.6 & 57.4 & 6.0 \\
\hline 36.2 & 57.0 & 6.8 \\
\hline
\end{tabular}

Fuentes: CELADE, 1987 a, p. 13; 1987 b, p. 39.
El Cuadro 1 pone en evidencia el carácter joven de las poblaciones de cada pais del istmo: todos ellos tienen un porcentaje elevado de su población concentrado en los primeros 15 aflos de vida. Incluso Costa Rica y Panamá, que por haber tenido un descenso de la fecundidad más pronunciado y precoz que los otros países del área presentan en el cuadro un porcentaje menor en las edades jóvenes, dicho rango de edad concentra más de un tercio de sus poblaciones respectivas. En los otros países el porcentaje es aún mayor.

\section{El impacto de los nacimientos ocurridos du-} rante el período 1980-2000

Es de sobra conocido el ciclo de vida del ser humano, que transcurre desde su nacimiento hasta su muerte, pasando por una serie de actividades desarrolladas entre esos dos momentos. En lo que respecta a este trabajo, vale la pena recordar que las personas nacidas en un momento dado, - con el devenir del tiempo- asistirán a la escuela, se incorporarán al mercado de trabajo y se reproducirán.

También es sabido que los nacimientos constituyen la principal fuente de incremento poblacional. En este trabajo se desea analizar cuál será la repercusión que tendrán los nacimientos ocurridos durante el periodo 1980-2000 sobre la estructura del conjunto de la población de cada país, así como sobre dos de sus subpoblaciones: aquella en edad escolar y la económicamente activa, con el consiguiente efecto sobre las futuras demandas de educación y empleo.

Se escogió el período antes mencionado no sólo porque en el momento actual estamos prácticamente a mitad del mismo, lo cual facilita el análisis; sino por otras dos razones: en primer lugar, porque es factible observar cuáles serán las consecuencias cuantitativas de esos nacimientos en un futuro cercano, por lo que el análisis puede resultar útil para la planificación a corto y mediano plazo; y, en segundo lugar, porque el lapso transcurrido entre el inicio y fin del período en cuestión es suficiente para que las personas nacidas durante el mismo hayan alcanzado una edad en la cual empiecen a incorporarse a las subpoblaciones indicadas. 


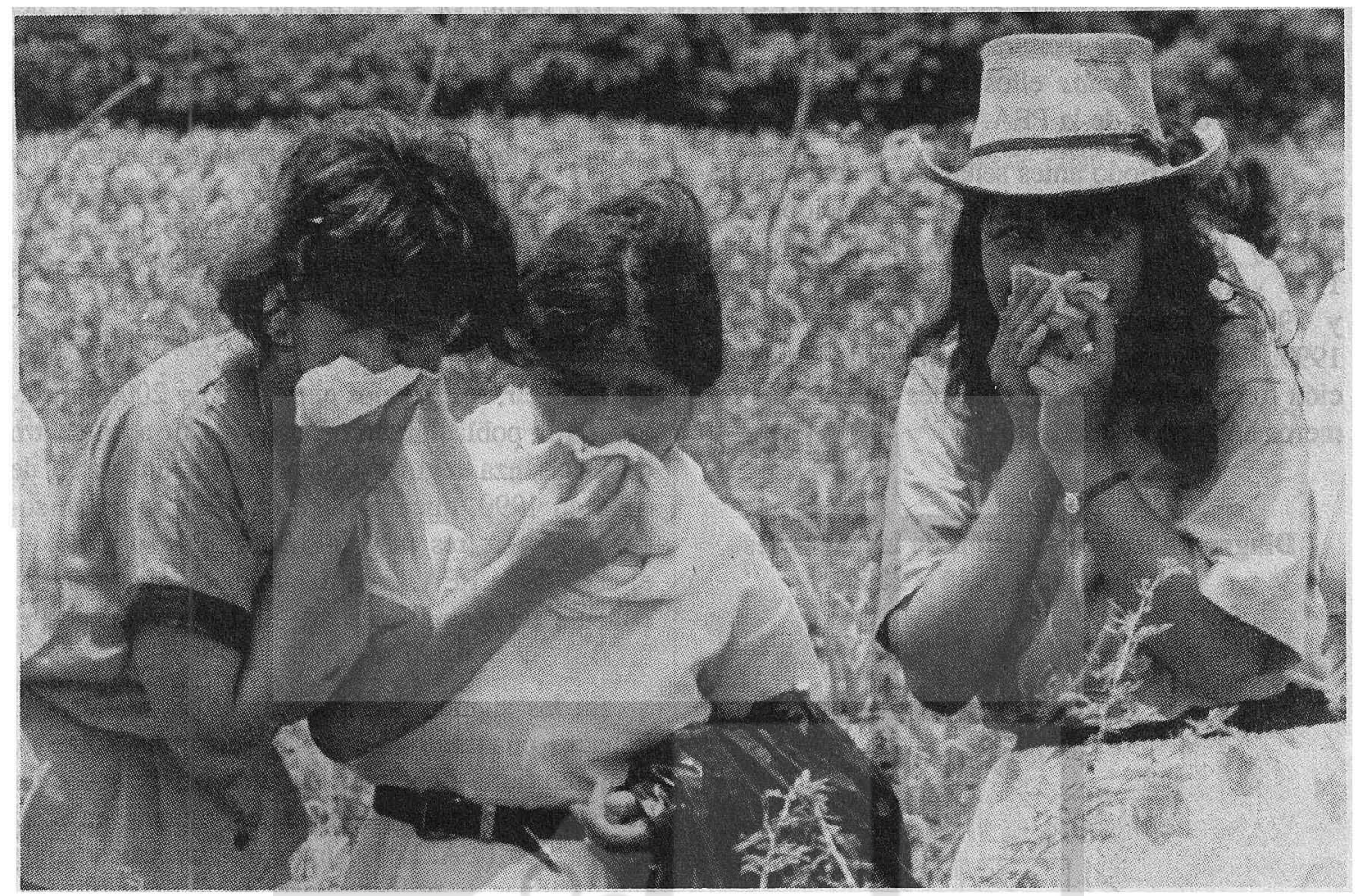

Con respecto a los límites de edad de las dos subpoblaciones a analizar, se comprenderá como "población en edad escolar" aquella que incluye las edades entre 5 y 24 años cumplidos, esperando abarcar dentro de ese rango la mayor parte de la población que se encuentra estudiando entre el nivel parvulario y el universitario. Por otro lado, se entenderá por "población económicamente acti$v a$ " (PEA) aquella productora de bienes y, o servicios con 10 o más años de edad. Estos límites de edad son los corrientemente utilizados en los instrumentos de recolección de información estadística en la mayor parte de países del tercer mundo.

Para facilitar el análisis, se utilizarán generaciones o "cohortes" quinquenales, de tal manera que pueda observarse gradualmente la entrada de cada una de ellas dentro de las dos subpoblaciones a estudiar. Por cohorte quinquenal se comprenderá a los sobrevivientes de los nacidos a lo largo de cinco años, los cuales evolucionarán juntos a lo largo de la vida, extinguiéndose gradualmente durante el transcurso de la misma, hasta que todos hayan perecido. Por ejemplo, se dirá "la cohorte 1980-1984", para señalar a todos los nacidos entre el 1 de enero de 1980 y el 31 de diciembre de 1984, cuya evolución podrá seguirse mientras avanzan en la vida: cuántos de ellos sobrevivirán a la mortalidad del primer año de vida, cuántos de esos sobrevivientes entrarán a la escuela, cuántos de los sobrevivientes formarán parte de la escuela, cuántos de los sobrevivientes formarán parte de la PEA y, al interior de ésta, cuántos lograrán un empleo, etc. Las generaciones nacidas en otros quinquenios (1985-1989, 1990-1994, etc.) seguirán su respectiva evolución de vida.

Como es natural, no todos los sobrevivientes de los nacidos en una misma cohorte quinquenal arriban simultáneamente a las edades adoptadas como limite inferior de las subpoblaciones en cuestión. Por ejemplo, si se toma la generación de niños nacidos entre 1980 y 1984, los venidos al mundo en 1980 estarán en edad para asistir a la escuela parvularia en 1985 , pero sus compañeros de cohorte nacidos en 1984 sólo podrán hacerlo a partir de 1989. Lo que sí puede asegurarse es que 
todos los de esa cohorte estarán en edad escolar en 1990. De igual manera, puede decirse que, a partir de 1995, todos ellos tendrán edades comprendidas dentro de la PEA.

Así, del periodo antes señalado se tendrán cuatro cohortes quinquenales: la de los nacidos entre 1980 y 1984 , la de los venidos al mundo entre 1985 y 1989, y la de quienes vendrán entre 1990 y 1994 y aquellos que lo hagan entre 1995 y 1999. La Figura 1 ayuda a comprender la evolución longitudinal de esas cohortes durante sus primeros años de vida.

\section{Figura 1}

Diagrama de la evolución de las cohortes nacidas entre 1980 y 2000

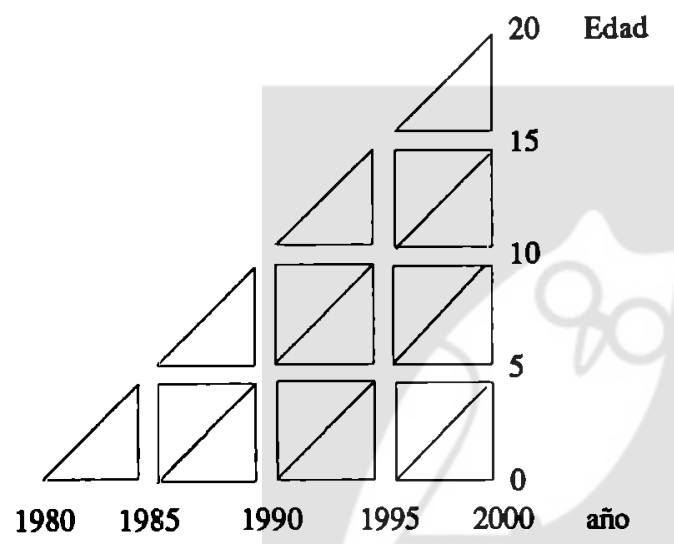

Como ya se dijo anteriormente, los miembros de la primera de esas cohortes que sobrevivan hasta 1990 habrán alcanzado, sin excepción alguna, la edad mínima para entrar al sistema de enseñanza: todos ellos tendrán entre 5 y 9 años cumplidos el año indicado. En 1995, habrá dos cohortes en edad de estar incorporadas a la escuela: la nacida entre 1980 y 1984 - la cual tendrá entonces entre 10 y 14 ańos cumplidos-y la de los nacidos entre 1985 y 1989, que contará con edades entre 5 y 9 ahios, también cumplidos.

El afio 2000 tendrá tres de esas cohortes en edad de asistir a la escuela: las dos ya mencionadas - las cuales para entonces tendrán entre 15 y 19 y 10 y 14 años de edad, respectivamentemás aquella de los nacidos entre 1990 y 1994, que tendrán entre 5 y 9 años cumplidos.
Tal como ya se mencionó antes, a partir del afio 1995 , la totalidad de los sobrevivientes de la primera de esas cohortes habrá alcanzado la edad inicial para ser considerado dentro de la PEA, cuando todos sus sobrevivientes tengan entre 10 y 14 años cumplidos. El año 2000 verá la incorporación de los nacidos entre 1985 y 1989, quienes alcanzarán el grupo de edad mencionado, mientras que, en ese af́o, la cohorte anterior ya habrá llegado a edades entre los 15 y 19 años cumplidos.

Es decir, los nacidos entre 1980 y 2000 impactarán a la población en edad de asistir a un centro de enseñanza regular solamente en un grupo de edad en 1990, mientras que en 1995 lo harán sobre dos, y en tres en el año 2000. Por su parte, en el caso de la PEA, esos nacimientos afectarán solamente un grupo de edad en 1995 y dos en el 2000.

En las siguientes subsecciones se analizará el impacto cuantitativo de estas cohortes sobre el conjunto de la población de cada país y sobre las dos subpoblaciones ya indicadas: aquella en edad escolar y la PEA. Los datos a utilizar proceden de estimaciones y proyecciones demográficas efectuadas por el Centro Latinoamericano de Demografia (CELADE, 1987), las cuales permiten analizar la evolución del conjunto de la población, así como la de aquella en edad escolar. Por su parte, los datos sobre la PEA proceden de otro boletín demográfico de la misma institución (CELADE, Boletín demográfico, No. 35).

\subsection{El impacto sobre el total de la población}

El Cuadro 2 permite observar la importancia porcentual que tienen las cuatro cohortes de nacidos entre 1980 y 1989 sobre los correspondientes grupos de edad del conjunto de la población de cada país o región, en cada uno de cuatro años específicos: $1985,1990,1995$ y 2000 . Como es natural, para cada uno de esos años, el cuadro sólo presenta cifras para los grupos de edad que proceden de las cuatro cohortes analizadas. Así, por ejemplo, en 1985 únicamente aparece el porcentaje del grupo $0-4$, pues para ese año, las personas que tenian 5 años o más correspondían a cohortes nacidas antes de 1980, que no son objeto de este trabajo. 


\section{Cuadro 2}

1985-2000: Porcentaje de la contribución de ciertos grupos de edad al total de la población de cada región o país en un año determinado

\begin{tabular}{|c|c|c|c|c|c|c|c|c|}
\hline \multirow[b]{2}{*}{ Año } & \multicolumn{3}{|c|}{ Grupo de edad } & \multirow[b]{2}{*}{$0-19$} & \multirow[b]{2}{*}{$0-4$} & \multicolumn{2}{|c|}{ Grupo de edad } & \multirow[b]{2}{*}{$0-19$} \\
\hline & $0-4$ & $\begin{array}{l}0-9 \\
\text { Istme }\end{array}$ & $0-14$ & & & $\begin{array}{l}0-9 \\
\text { Cent }\end{array}$ & $\begin{array}{c}0-14 \\
\mathrm{ca}\end{array}$ & \\
\hline 1985 & 16.9 & & & & 17.2 & & & \\
\hline 1990 & 16.3 & 30.6 & & & 16.6 & 31.2 & & \\
\hline 1995 & 15.6 & 29.5 & 41.9 & & 16.0 & 30.1 & 42.7 & \\
\hline \multirow[t]{2}{*}{2000} & 14.8 & 28.3 & 40.4 & 51.1 & 15.1 & 28.9 & 41.1 & 52.0 \\
\hline & \multicolumn{4}{|c|}{ Guatemala } & & \multicolumn{3}{|c|}{ El Salvador } \\
\hline 1985 & 18.0 & & & & 16.5 & & & \\
\hline 1990 & 17.5 & 32.5 & & & 16.0 & 30.5 & & \\
\hline 1995 & 16.8 & 31.5 & 44.3 & & 16.0 & 29.9 & 42.5 & \\
\hline \multirow[t]{2}{*}{2000} & 16.0 & 30.3 & 42.9 & 53.9 & 15.6 & 29.5 & 41.5 & 52.4 \\
\hline & \multicolumn{3}{|c|}{ Honduras } & & & \multicolumn{2}{|c|}{ Nicaragua } & \\
\hline 1985 & 17.8 & & & & 18.5 & & & \\
\hline 1990 & 17.0 & 31.9 & & & 17.8 & 33.0 & & \\
\hline 1995 & 16.1 & 30.5 & 43.2 & & 16.8 & 31.7 & 44.6 & \\
\hline \multirow[t]{2}{*}{2000} & 14.9 & 28.7 & 41.2 & 52.2 & 15.7 & 29.9 & 42.7 & 53.7 \\
\hline & & Cost: & & & & Pana & & \\
\hline 1985 & 13.8 & & & & 12.9 & & & \\
\hline 1990 & 13.1 & 25.2 & & & 12.3 & 23.9 & & \\
\hline 1995 & 11.9 & 23.6 & 34.5 & & 11.6 & 22.8 & 33.2 & \\
\hline 2000 & 10.9 & 21.7 & 32.3 & 42.2 & 10.7 & 21.3 & 31.5 & 40.9 \\
\hline
\end{tabular}

Fuente: CELADE, Boletín demográfico, No. 40, 1987.

En 1990 hay datos sobre los grupos $0-4$ y $0-9$, pues tanto el grupo 0-4 como el 5-9 -que conjuntamente constituyen el segundo de esos grupos- pertenecen a cohortes nacidas durante el período analizado. En 1995 también figura el porcentaje del grupo 0-14, y en el año 2000 se incluye al grupo 0-19 por la misma razón.

El cuadro muestra que en 1990 los sobrevivientes de los nacimientos ocurridos durante la década de los años ochenta representaban casi un tercio de la población total del istmo, así como la de cada país de la región. En el caso de Costa Ri- ca y Panamá - que ya se vio que tienen las poblaciones más "envejecidas" del área-, su importancia porcentual es menor, pero de todas formas ellos constituyen alrededor de la cuarta parte del conjunto de sus respectivas poblaciones, lo cual es un porcentaje significativo. Es decir, los sobrevivientes de los nacimientos ocurridos durante la década de los ochenta, actualmente constituyen un porcentaje considerable de la población de cada país centroamericano.

Dentro de sólo cuatro años, en 1995, el impacto del conjunto de las tres cohortes de nacidos 
hasta ese momento -1980-1994 representará una cifra cercana a la mitad del conjunto de la población de cada país: alrededor del 42 por ciento de la población del istmo y de Centroamérica, habiendo países con valores superiores a esa cifra como Guatemala y Nicaragua, casi con el 45 por ciento-y otros inferiores -como Costa Rica y Panamá-, pero que aún en este caso representarán por lo menos un tercio del total de la población.

En el caso del año 2000, del cual nos separa ya menos de una década, sólo los sobrevivientes de la población que nacerá en el transcurso de los años noventa constituirán más de la cuarta parte de la población del istmo (28.3 por ciento) y de Centroamérica (28.9 por ciento), con valores extremos, oscilando alrededor del 30 por ciento en Guatemala, El Salvador y Nicaragua, por una parte, y del 22 por ciento en Costa Rica y Panamá, por la otra. En este año, esta población tendrá entre los 0 y 9 años cumplidos de edad.

Ese mismo año, los sobrevivientes de las cuatro cohortes analizadas formarán más de la milad de la población del istmo (51.1 por ciento) y de Centroamérica (52.0 por ciento), con oscilaciones extremas desde alrededor del 54 por ciento en Guatemala y Nicaragua, hasta aproximadamente el 42 por ciento en Costa Rica y Panamá. Vale la pena subrayar que en dicho año, más de la mitad de la población de la región estará constituida por sobrevivientes de las personas nacidas en las décadas del ochenta y del noventa; es decir, por individuos que actualmente son niños o que aún no han nacido.

Esto demuestra la importancia que esta población infantil debería tener en la planificación socio-económica de los países de la región en el momento actual, pues dentro de apenas nueve años lo que los países hagan o dejen de hacer por ellos estará beneficiando o afectando a más de la mitad de los habitantes de Centroamérica.

\subsection{El impacto sobre ciertas subpoblaciones}

La presente sección trata de señalar el impacto cuantitativo que tendrán los nacimientos provenientes de las cohortes analizadas sobre dos sub- poblaciones: aquella en edad escolar y la económicamente activa.

\subsubsection{El impacto sobre la subpoblación en edad escolar}

Para estudiar este impacto se utilizará el Cuadro 3, en el cual se incluye el número de sobrevivientes de los nacidos entre 1980 y 1999, y su importancia porcentual sobre el conjunto de la población en edad escolar, para los años 1990, 1995 y 2000 .

En 1990, sólo los sobrevivientes de una de las cohortes - la nacida entre 1980 y 1984 - estarán en edad para asistir a la escuela. Es decir, del total de la población en edad escolar en 1990, un sólo grupo de edad quinquenal proviene de las cohortes analizadas: el de 5-9 años cumplidos. Sin embargo, ese único grupo constituye, en el conjunto del istmo, casi un tercio de dicha subpoblación (30.4 por ciento), con valores un poco menores en Costa Rica y Panamá, pero incluso en ambos casos dichos valores son superiores a la cuarta parte de las subpoblaciones respectivas.

En 1995, ya serán dos grupos de edad (5-9 y 10-14) los que provendrán de las cohortes en cuestión. Es decir, dentro de sólo cuatro años esas dos cohortes constituirán más de la mitad de la población escolar (56.6 por ciento en el istmo). Incluso los países con población más "envejecida", como Costa Rica y Panamá, tendrán esta misma característica: el 55.2 y el 52.1 por ciento, respectivamente.

Finalmente, en el año 2000, los tres grupos quinquenales que provendrán de las cohortes analizadas (5-9, 10-14 y 15-19 años) compondrán más de las tres cuartas partes de la población estudiantil de ese año: el 79.5 por ciento en el istmo, con valores extremos en Nicaragua (80.5 por ciento) y Panamá ( 77.0 por ciento).

En breve, los sobrevivientes de las cohortes de nacimientos ocurridos en los últimos diez años, así como las que se incorporarán en el transcurso de la presente década, tienen desde este momento un impacto muy importante en la estructura educativa de la región, la cual —en base a lo mostrado por el Cuadro 3 - no hará más que incremen- 


\section{Cuadro 3}

Centroamérica, 1990-2000: número de sobrevivientes de los nacidos entre 1980 y 2000 que se encuentran en edad escolar y su importancia porcentual sobre el total de dicha población, por región y país

Aflo Sobrevivientes de las cohortes que el año $\mathrm{X}$ se encuentran en edad escolar

\begin{tabular}{lcccccccc}
\hline $\mathbf{X}$ & $\begin{array}{c}\text { Efectivos } \\
\text { Istmo }\end{array}$ & $\%$ & $\begin{array}{c}\text { Efectivos } \\
\text { Centroamérica }\end{array}$ & $\%$ & $\begin{array}{c}\text { Efectivos } \\
\text { Guatemala }\end{array}$ & $\%$ & $\begin{array}{c}\text { Efectivos } \\
\text { El Salvador }\end{array}$ & $\%$ \\
\hline 1990 & 4143255 & 30.4 & 3864721 & 30.7 & 1380583 & 31.7 & 763324 & 29.4 \\
1995 & $\mathbf{8 7 0 9 0 2 5}$ & 56.6 & 8136040 & 56.9 & 2919500 & 57.9 & 1574683 & 55.0 \\
2000 & 13701686 & 79.5 & 12826741 & 79.7 & 4626869 & 80.3 & 2481271 & 78.7 \\
& Honduras & & Nicaragua & & Costa Rica & & Panamá & \\
1990 & 765799 & 30.8 & 588959 & 31.7 & 366056 & 28.7 & 278534 & 26.4 \\
1995 & 1619253 & 56.8 & 1260860 & 58.2 & 761744 & 55.2 & 572985 & 52.1 \\
2000 & 2554251 & 80.0 & 2003709 & 80.5 & 1160641 & 77.8 & $\mathbf{8 7 4 9 4 5}$ & 77.0 \\
\hline
\end{tabular}

Fuente: CELADE, Boletín demograffico Nos. 38 y 40.

tarse de manera todavía más significativa en el transcurso de la presente década.

3.2.2. El impacto sobre la subpoblación económicamente activa

Antes de analizar el contenido del Cuadro 4, referente a la Población Económicamente Activa (PEA), conviene tener presente que ésta se encuentra influida tanto por el contingente de personas en edad de trabajar como también por las tasas de actividad, es decir, por la participación de ese contingente en tareas socialmente útiles en la producción de bienes o servicios para la sociedad, ya sea que se encuentren ocupadas o no, durante el período de referencia utilizado por el instrumento de recolección de datos que se haya empleado para obtener esa información.

También es importante subrayar el hecho de la imperfección que todavía existe en la definición de la PEA. Este problema es mayor en el caso de ciertas subpoblaciones, por ejemplo, en la del trabajo femenino - sobre todo en las zonas rurales-

\section{Cuadro 4}

Centroamérica, 1995 y 2000: número de sobrevivientes de los nacidos entre 1980 y 2000 participantes en la PEA y su importancia porcentual en esa población, por región y país

\begin{tabular}{|c|c|c|c|c|c|c|c|c|}
\hline \multirow{2}{*}{$\frac{\text { Año }}{X}$} & \multicolumn{8}{|c|}{ Sobrevivientes de las cohortes que el año X participarán en la PEA } \\
\hline & $\begin{array}{c}\text { Efectivos } \\
\text { Istmo }\end{array}$ & $\%$ & $\begin{array}{c}\text { Efectivos } \\
\text { Centroamérica }\end{array}$ & $\%$ & $\begin{array}{c}\text { Efectivos } \\
\text { Guatemala }\end{array}$ & $\%$ & $\begin{array}{c}\text { Efectivos } \\
\text { El Salvador }\end{array}$ & $\%$ \\
\hline $\begin{array}{l}1995 \\
2000\end{array}$ & $\begin{array}{r}430862 \\
2107692\end{array}$ & $\begin{array}{r}3.8 \\
15.7\end{array}$ & $\begin{array}{r}420372 \\
2021794\end{array}$ & $\begin{array}{r}4.0 \\
16.4\end{array}$ & $\begin{array}{l}167969 \\
715009\end{array}$ & $\begin{array}{r}5.2 \\
18.7\end{array}$ & $\begin{array}{l}119581 \\
525900\end{array}$ & $\begin{array}{r}4.4 \\
16.5\end{array}$ \\
\hline & Honduras & & Nicaragua & & Costa Rica & & Panamá & \\
\hline $\begin{array}{l}1995 \\
2000\end{array}$ & $\begin{array}{r}62311 \\
369845\end{array}$ & $\begin{array}{r}3.3 \\
16.5\end{array}$ & $\begin{array}{r}49258 \\
245381\end{array}$ & $\begin{array}{r}3.5 \\
14.5\end{array}$ & $\begin{array}{r}21253 \\
165659\end{array}$ & $\begin{array}{r}1.8 \\
12.1\end{array}$ & $\begin{array}{l}10490 \\
85898\end{array}$ & $\begin{array}{l}1.1 \\
7.9\end{array}$ \\
\hline
\end{tabular}

Fuente: CELADE, Boletín demográfico No. 35. 
y, en el tema que nos ocupa, la incorporación precoz a la actividad económica de una cantidad considerable de la población centroamericana. Debido a lo anterior, es muy posible que la medición del primer grupo quinquenal de la PEA -10 a 14 años - padezca de una subestimación considerable en relación a la realidad.

El impacto ejercido por las cohortes nacidas entre 1980 y 2000 sobre el conjunto de la PEA de cada país en 1955 se limita a un grupo de edad, el de 10 a 14 años, que corresponde a las personas nacidas entre 1980 y 1985, y que para ese año ya habrán alcanzado el límite inferior de edad de la PEA.

Considerando el obstáculo de subestimación mencionado arriba, además del que se deriva del hecho de que no toda la población forma parte de la PEA y, finalmente, el proveniente del factor que en esta subpoblación se toma en consideración un amplio grupo de edad (10 años y más), no es de extrañar que el ya citado único grupo de edad tenga una influencia limitada: 3.8 por ciento en el conjunto del istmo, como puede constatarse en el Cuadro 4.

Sin embargo, apenas cinco años después —en el año $2000^{-}$, a pesar de que las cohortes en cuestión sólo estarán contribuyendo con dos grupos quinquenales a la PEA (10-14 y 15-19), éstas ya representarán el 15.7 por ciento del conjunto de esa subpoblación en el istmo, con valores extremos que van desde el 18.7 por ciento en Guatemala hasta el 7.9 por ciento en Panamá. Es evidente que el aporte a la PEA de las generaciones analizadas se incrementará mucho más después del año ya indicado, pero ese periodo escapa al fijado para este trabajo.

Por lo tanto, también en este caso puede observarse que desde fines de década en curso, los sobrevivientes de los nacimientos ocurridos en el período analizado irán convirtiéndose de manera acelerada en un componente cada vez más importante en términos cuantitativos - tanto a nivel de efectivos como a nivel porcentual一, subrayando con ello la necesidad que tienen los paises del istmo de preparar estos niños de manera que puedan llegar a convertirse en mano de obra calificada que contribuya al desarrollo socio-económico del área y, más importante aún, la necesidad de los países para prepararse a ofrecer a esa población un empleo digno.

\section{Conclusiones}

Las condiciones socio-económicas en las cuales se desarrollan actualmente las sociedades centroamericanas son muy difíciles, como puede constatarse en el Cuadro 5. De no efectuarse cambios sustanciales en la estructura y en el modelo de desarrollo de esas sociedades, los nuevos contingentes poblacionales que vayan incorporándose a ellas no sólo se enfrentarán a condiciones aún más deterioradas, sino que su incremento contribuirá a volver aún más difícil de resolver la problemática que las afecta.

El cuadro en referencia destaca que en la mayoria de los países centroamericanos, el porcentaje de analfabetismo todavía es alto, la mortalidad infantil se sitúa a nivel intermedio al compararla con otras regiones del mundo, la mayoría de la población continúa muriendo por causas que podrian evitarse con relativa facilidad por medio de la medicina preventiva - aunque la violencia también cobra un elevado porcentaje de vidas-, las condiciones de vivienda indican un alto nivel de hacinamiento, el desempleo abierto y disfrazado afecta a un elevado porcentaje de la PEA de cada uno de esos países, y el porcentaje de pobres comprende alrededor de los dos tercios de las poblaciones respectivas.

Si en el momento actual no se adoptan decisiones firmes, el futuro a mediano plazo de la población de la región - para no hablar del largo término-se anuncia cada vez menos promisorio.

Si esas transformaciones no llegaran a efectuarse, la población que acaba de nacer -así como la que está por venir al mundo- se encontrará viviendo en una región que no le ofrecerá más expectativa que la de tratar de emigrar de ella. Por el contrario, si los países lograr abrir una brecha que permita vislumbrar la luz al final del túnel, el futuro de Centroamérica podría consolidarse gradualmente.

En este trabajo se ha analizado el impacto 


\section{Cuadro 5}

Algunos indicadores socio-económicos de la población centroamericana, por país

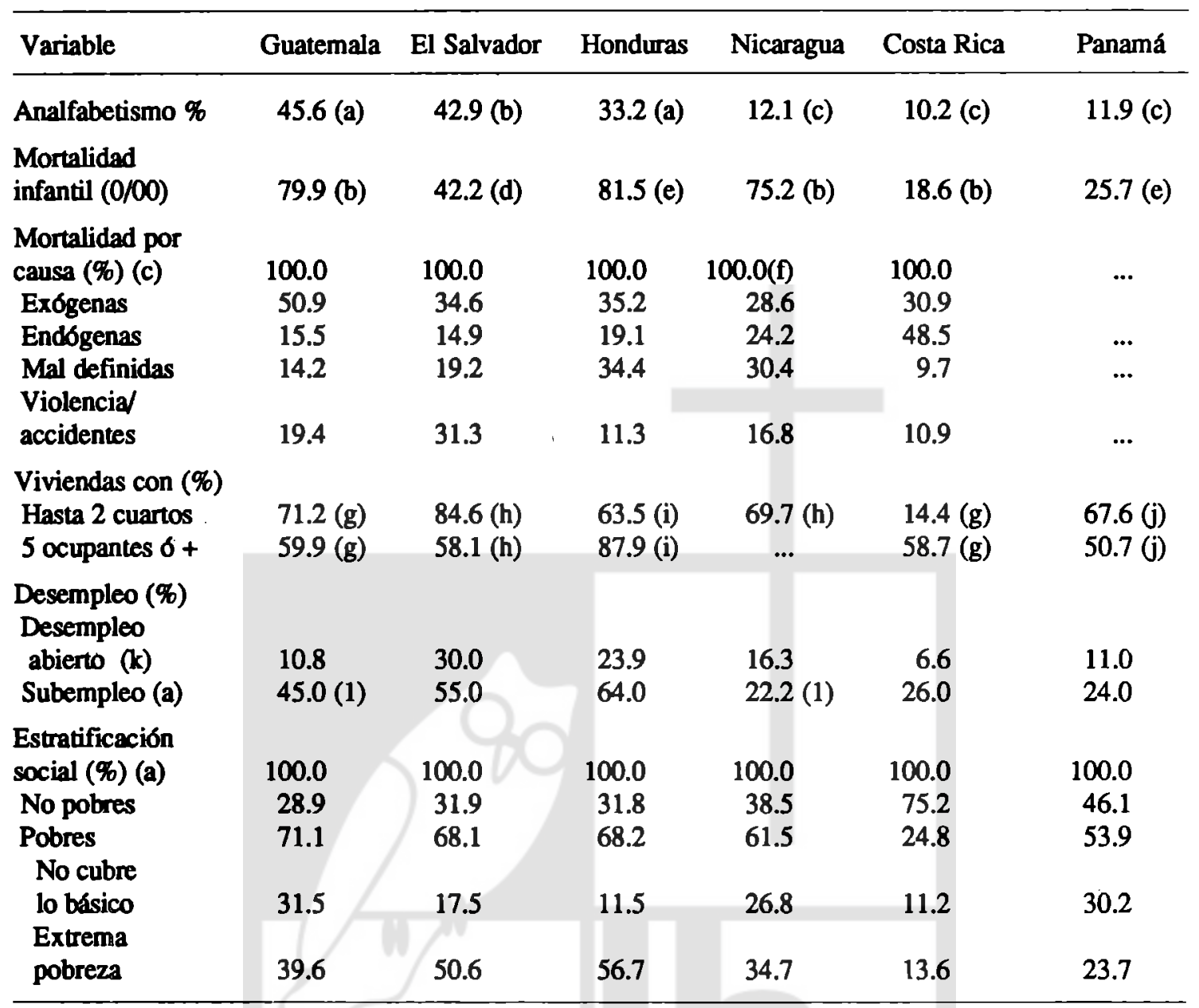

... No disponible
(a) 1980
(e) $1980-1985$
(b) 1983
(f) 1978
(c) 1981
(g) 1973
(d) 1982
(h) 1971
(i) 1974
(j) 1970
(k) 1984
(l) 1985

Fuenses: -Gallardo y Lopez, 1986, pp. 158, 189, 200 y 214; WHO, 1981 (Cuadro 7B), 1984 (Cuadro 12), 1985. (Cuadro 13) y 1986 (Cuadros 1 y 2); CELADE, 1987a, p. 109.

cuantitativo de las generaciones nacidas entre 1980 y 2000 sobre la población total, así como también sobre las poblaciones en edad escolar y sobre la PEA, por considerar que ambas constituyen elementos importantes para el desarrollo de la región. La calificación técnica o profesional de la población resulta imprescindible si se desea ese desarrollo, y proporcionar empleo a la población activa no sólo tiene como consecuencia el evidente beneficio económico individual y colectivo, sino que también aporta imponderables ventajas para el desarrollo social.

Si las sociedades del istmo centroamericano 
lograran influir positivamente sobre dichas generaciones, por la sola importancia cuantitativa que ellas tienen $-y$ que ha sido subrayada en este trabajo-, ellas podrían introducir un sesgo favorable en el conjunto de la población respectiva, así como en las dos subpoblaciones enfocadas, y todo ello en un futuro inmediato, con el consiguiente efecto sobre el conjunto de la sociedad.

Sin embargo, falta aún determinar las características cualitativas que ese accionar deberia tener, como para que pueda dar frutos verdaderamente efectivos. Sin garantizar el contenido cualitativo de las transformaciones necesarias, la importancia cuantitativa de las cohortes analizadas no podrá contribuir sustancialmente a la solución de la actual problemática socio-económica, sino que más bien a la complicación de la misma.

\section{Bibliografía}

Centro Latinoamericano de Demografía, CELADE, Boletín demográfico, No. 35, Santiago, Chile.

Centro Latinoamericano de Demografía, CELADE, Bo- letín demográfico, año XTX, No. 38, Santiago, Chile, 1986.

Centro Latinoamericano de Demografía, CELADE, Boletín demográfico, Año XX, No. 40, Santiago, Chile, 1987a.

Centro Latinoamericano de Demografía, CELADE, América Latina en el año de los 5.000.000.000, Santiago, Chile, 1987b.

Comisión Económica para América Latina y el Caribe, CEPAL, Anuario estadístico de América Latina y el Caribe, Edición 1987, Santiago, Chile, 1987.

Gallardo, M. y López, J., Centroamérica. La crisis en cifras, Ed. IICA-FLACSO, San José, Costa Rica, 1986.

Nations-Unies, Annuaire démographique, 1982, 34éme édition, New York, Estados Unidos, 1984.

World Health Organization, WHO, 1981 World Health Statistics Annual, Ginebra, Suiza, 1981.

World Health Organization, WHO, 1984 World Health Statistics Annual, Ginebra, Suiza, 1984.

World Health Organization, WHO, 1985 World Health Statistics Annual, Ginebra, Suiza, 1985.

World Health Organization, WHO, 1986 World Health Statistics Annual, Ginebra, Suiza, 1986. 